\title{
Reduced Order Modeling of the Pressure Distribution over the AGARD 445.6 Wing
}

\author{
Yanal Issac* \\ Georgia Institute of Technology, Atlanta, Georgia, 30332, USA \\ Walter A. Silva ${ }^{\dagger}$ \\ NASA Langley Research Center, Hampton, Virginia, 23681, USA \\ Dimitri Mavris ${ }^{\ddagger}$ \\ Georgia Institute of Technology, Atlanta, Georgia, 30332, USA
}

\begin{abstract}
A reduced order model (ROM) has been developed for the pressure distribution over the AGARD 445.6 wing using a computational fluid dynamics (CFD) code, FUN3D version 12.9. Modal step responses of the inviscid, unsteady aerodynamic system are computed using the FUN3D code. Using proper orthogonal decomposition (POD), the modal step responses are decomposed into a set of pressure modes and a set of generalized coordinates associated with every pressure mode. An appropriate subset of the generalized coordinates is then transformed into state-space form. The unsteady aerodynamic state-space ROM could then be combined with a state-space model of the structure to create an aeroelastic simulation in MATLAB. The aerodynamic model is orders of magnitude faster than the high-order solution procedure developed by the use of traditional fluid and structural solvers enabling flutter, aeroservoelasticity analysis, and optimization at an earlier stage of the fixed wing aircraft design process.
\end{abstract}

\section{Nomenclature}

$\beta \quad$ step input scaling parameter

$\Phi \quad$ proper orthogonal decomposition reduced-order mapping matrix

$A, B, C \quad$ state-space matrix operators

$E \quad$ relative pressure error

$H \quad$ Hankel matrix

$h_{t} \quad$ impulse response

$P, \hat{P} \quad$ full- and reduced-order discrete pressure variables

$P_{0} \quad$ base pressure solution

$P_{\mathrm{FUN} 3 \mathrm{D}} \quad$ predicted pressure distribution via FUN3D

$P_{\mathrm{ROM}} \quad$ predicted pressure distribution via ROM

$S \quad$ snapshot matrix of offset pressure distributions

$s_{t} \quad$ step response

$u \quad$ system input

$x \quad$ system state variables

y system response

*Ph.D. Candidate, School of Aerospace Engineering, Student Member AIAA

†Senior Research Scientist, Aeroelasticity Branch, Associate Fellow AIAA.

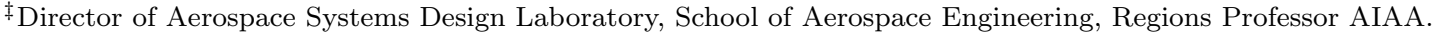




\section{Introduction}

$\mathrm{T}$ HE application of reduced order modeling (ROM) techniques to aeroelastic systems is an active area of research, motivated by the desire for faster algorithms that are well suited to the design environment for aircraft. For example, transonic, fluid-structure interaction is a particular application of interest to both external and internal aerodynamicists because moving shock waves in the flow necessitate high-fidelity numerical flow solvers that are too cumbersome for iterative design analysis. Regardless of the application, when nonlinearities are present in either the flowfield or the structure, established order reduction methods that rely on linearized dynamics are of little use. Volterra methods ${ }^{1}$ and proper orthogonal decomposition ${ }^{2,3}$ (POD) are two of the more prevalent ROM techniques well-suited to nonlinear dynamics. ${ }^{4-8}$ Traditionally, Volterra methods have relied on generalized aerodynamic forces (GAFs) to couple fluid forcing terms to structural modes. The combining of POD with Volterra methods is a way to replace the GAF terms with a physics-based fluid ROM that can potentially produce nonlinear aerodynamic forcing.

In this paper, we present a method for creating a reduced order model of the pressure distribution over the AGARD 445.6 wing from CFD data obtained using the FUN3D code. The flow is assumed to be inviscid with a constant free-stream Mach number and angle of attack. We present two cases, one at Mach 0.8 and another at Mach 1.1, both of which held the angle of attack at $0^{\circ}$.

\section{AGARD 445.6 Wing}

The AGARD 445.6 Aeroelastic Wing has been used extensively by several authors to validate computational methods. Although the aeroelastic behavior of this wing is fairly benign (weakly nonlinear), the aeroelastic data from the flutter test of this wing provides a good starting point for validation of computational techniques. The wing is a 45-degree swept-back wing with an NACA 65A004 airfoil section, panel aspect ratio of 1.65 , and a taper ratio of 0.6576 . The shape of the first four structural modes for this wing are presented in Figure 1. The modes are first bending, first torsion, second bending, and second torsion. The corresponding modal frequencies in vacuo are 9.60, 38.2, 48.35, and $91.54 \mathrm{~Hz}$.

\section{ROM Formulation}

This section introduces the proper orthogonal decomposition (POD) and overviews the eigensystem realization algorithm (ERA). In addition, we fully develop the POD-ERA approach and the synthesis of aeroelastic ROMs.

\section{III.A. ROM Development Process}

An outline of the ROM development process is as follows:

1. Implementation of step response technique into the aeroelastic CFD code.

2. Computation of the step responses for each mode of an aeroelastic system using the aeroelastic CFD code.

3. Dimensionality reduction of the step responses from step 2 using proper orthogonal decomposition.

4. Impulse responses are computed from the generalized coordinates in step 3 and are fed into the ERA.

5. Evaluation/validation of the state-space modes in step 4.

\section{III.B. Proper Orthogonal Decomposition}

Proper orthogonal decomposition is a technique used to decompose vector and scalar fields into a weighted sum of basis functions or modes. The modes represent the spatial component of the decomposed data while the weights represent the temporal component of the data. A detailed description of POD applied to spatially discretized flowields can be found in Ref. 9. A summary of POD as it applies to a spatially discretized scalarfield follows.

A POD reduced-order mapping $\Phi$ is constructed by the collection of observations of the solutions $P(t)-P_{0}$ at different time intervals throughout the time integration of the full-system dynamics. These observations 


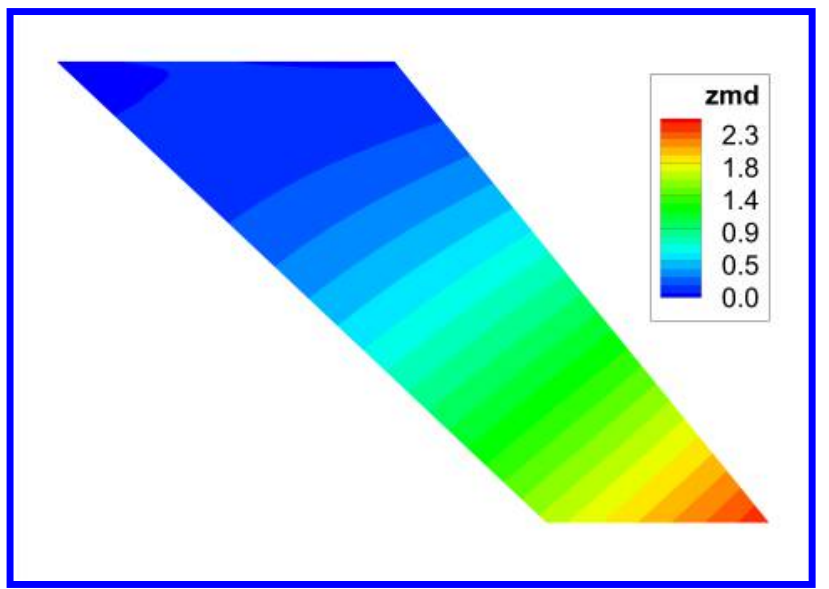

(a) First structural mode.

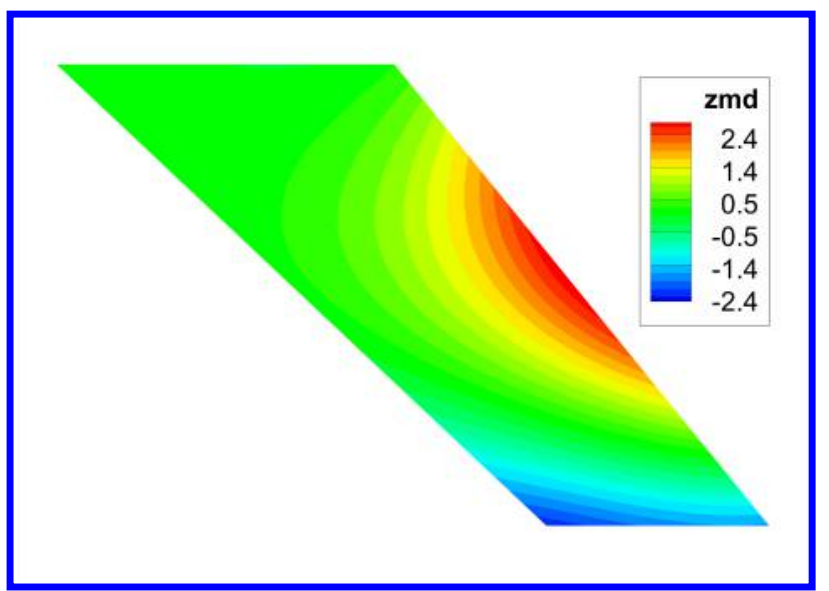

(c) Third structural mode.

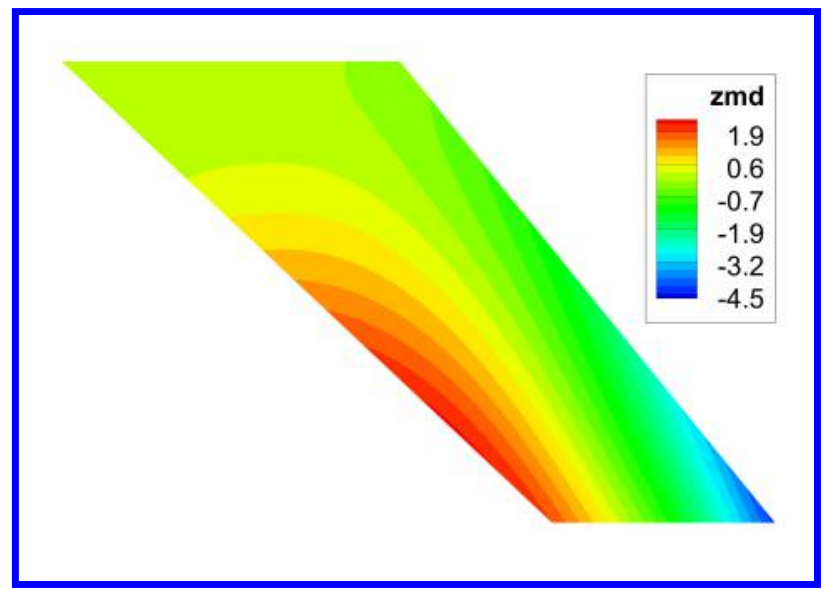

(b) Second structural mode.

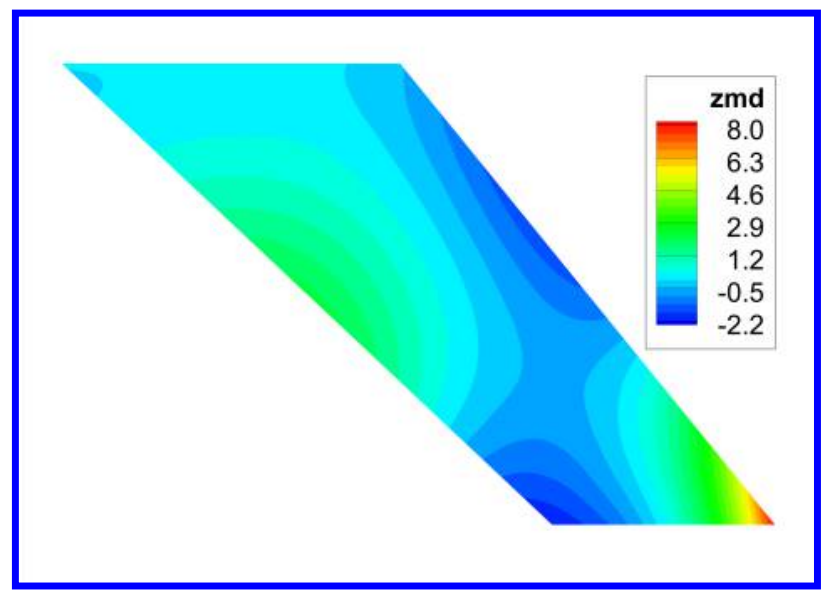

(d) Fourth structural mode.

Figure 1. The first four structural modes of the AGARD 445.6 wing where ' $z m d$ ' is the modal deflection in the z-direction.

are called snapshots ${ }^{9}$ and are generally collected to provide a good variety of pressure dynamics that minimize linear dependence. The snapshot generation procedure is sometimes referred to as POD training. ${ }^{9}$ Snapshots are compiled columnwise into a snapshot matrix $S$. Next, the eigenvectors $V$ are obtained from $S^{\top} S . S$ and $V$ produce a linear transformation $\Phi$ between the full-order solution $P$ and the reduced-order solution $\hat{P}$ :

$$
\begin{gathered}
P[n] \approx P_{0}+\Phi \hat{P}[n], \\
\Phi=S V .
\end{gathered}
$$

The reduced-order variable $\hat{P}[n]$ represents deviations of $P[n]$ from a base solution $P_{0}$. The subtraction of $P_{0}$ will result in zero-valued boundaries for the POD modes wherever constant boundary conditions occur on the domain.

\section{III.C. Eigensystem Realization Algorithm}

The ERA method ${ }^{10}$ identifies a discrete, linear, time-invariant state-space realization of the form

$$
\begin{gathered}
x[n+1]=A x[n]+B \beta u[n], \\
y[n]=C x[n],
\end{gathered}
$$

by the use of data from a complete ensemble of impulse responses. The term $\beta$ is a scaling parameter that is necessary to calibrate the forcing amplitude when the impulse is other than unity. Theoretically, $\beta$ should be the inverse of the impulse amplitude and its inclusion allows more freedom when the full system is impulsed. 
Initial state responses can be used in lieu of impulse responses, but we only consider impulse response data in this overview for simplicity. The system realization procedure takes measurement data $y[n]$ from the free response of the system and produces a minimal state-space model $A, B$, and $C$ such that functions $y$ are accurately reproduced.

The free pulse response of linear, time-invariant, discrete systems is given by a function known as the Markov parameter,

$$
y_{m}[n]=C A^{n-1} B .
$$

The superposition principle states that a system response to any arbitrary input can be obtained from a linear combination of impulse responses from that system. The generalized Hankel matrix of impulse responses is related to the Markov parameter by the superposition principle. The Hankel matrix is formed by windowing the impulse response data. A total of $K$ data points are provided at discrete time steps $n=1, \ldots, K$, and the $r \times s$ matrix $H_{r s}$ is formed as follows:

$$
H_{r s}^{n-1}=\left[\begin{array}{ccc}
y_{m}[n] & \ldots & y_{m}[n+s-1] \\
y_{m}[1+n] & \ldots & y_{m}[1+n+s-1] \\
\vdots & \vdots & \vdots \\
y_{m}[r-1+n] & \ldots & y_{m}[r-1+n+s-1]
\end{array}\right],
$$

where $s$ is the total size of the data window, and $r$ is the number of time steps used to shift the data window. The choice of $r$ and $s$ is arbitrary as long as $r+s+n \leq K+2$. The ERA method eliminates redundant data by the use of singular value decomposition (SVD) on $H_{r s}^{0}$,

$$
H_{r s}^{0}=P D Q^{\top} .
$$

Unwanted state dimensionality is eliminated by truncation of the elements of $P, D$, and $Q$ associated with very small singular values of $H_{r s}^{0}$. The number of states is reduced to a minimal number $q$. The number of observations $p$ and the number of forcing terms $m$ are known from the problem formulation. The dimension of the Markov parameter $y_{m}[n]$ is $p \times m$. Algebra is used to recast Equation (5) in terms of the time shifted Hankel matrix $H_{r s}^{1}$ and the elements $P, D$, and $Q$. The state-space realization flows from this manipulation and is as follows:

$$
\begin{gathered}
A=D^{-\frac{1}{2}} P^{\top} H_{r s}^{1} Q D^{-\frac{1}{2}}, \\
B=D^{\frac{1}{2}} Q^{\top} E_{m}, \\
C=E_{p}^{\top} P D^{\frac{1}{2}} .
\end{gathered}
$$

$E_{p}^{\top}$ and $E_{m}^{\top}$ are defined as

$$
\begin{gathered}
E_{p}^{\top}=\left[\begin{array}{llll}
I_{p}, & 0_{p}, & \ldots, & 0_{p}
\end{array}\right], \\
E_{m}^{\top}=\left[\begin{array}{llll}
I_{m}, & 0_{m}, & \ldots, & 0_{m}
\end{array}\right],
\end{gathered}
$$

where $0_{p}$ and $0_{m}$ are the null matrices of order $p$ and $m$, respectively, and $I_{p}$ and $I_{m}$ are the identity matrices of order $p$ and $m$.

Because the discrete time step $\delta t=t_{k+1}-t_{k}$ is constant, the continuous form of the discrete state-space realization is easily obtained. The continuous form, shown hereafter, absorbs the scaling parameter $\beta$ into the matrix $B$ and may require additional state dimensionality when the discrete realization has real, negative poles:

$$
\begin{gathered}
x[n+1]=A x[n]+B u[n], \\
y[n]=C x[n] .
\end{gathered}
$$




\section{Results}

Figure 2 shows two of the snapshots obtained from a simulation at Mach 0.8. Figure 2(a) shows the pressure distribution over the AGARD wing when no structural deformation is present, this is in contrast to Figure 2(b) thatshows the pressure distribution over the AGARD wing when a step input is applied to all of the structural modes. Step or impulse inputs are usually used to excite all the frequencies of a system, however, a step input is easier to implement in FUN3D, and its responses are more numerically stable. The pressure distribution over the undeformed wing is used to create the base solution vector $P_{0}$.

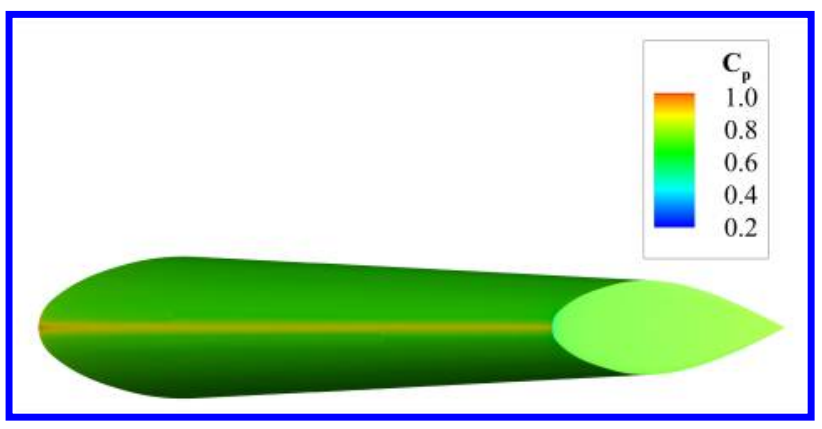

(a) AGARD 445.6 wing without any input applied to the structural modes. The data from this snapshot is arranged in the column vector $P_{0}$.

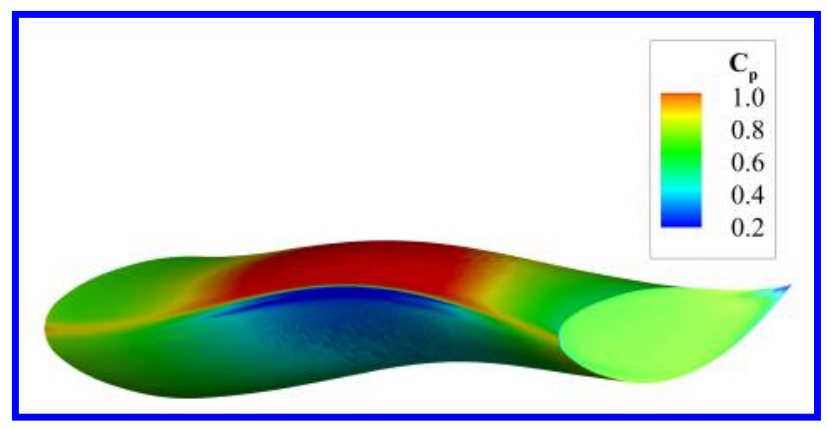

(b) A specific snapshot showing the pressure distribution over the AGARD 445.6 Wing when a step input is applied to all four structural modes.

Figure 2. Wing shape and pressure distribution over the wing before and after the step input is applied to the structural modes. Note that the AGARD 445.6 wing is a very thin airfoil and the figure is not to scale, a visual distortion is applied in the z-direction for the aid of visualization.

The base solution $P_{0}$ is then used to offset the remainder of the pressure distributions in the subsequent time steps obtained from the simulation. Figure 2(b) shows the pressure distribution over the deformed wing before subtracting the base solution $P_{0}$. The offset pressure distributions, snapshots, are arranged into columns and concatenated together to form the snapshot matrix $S$. As discussed previously, applying the POD to the snapshot matrix $S$, results in decomposing the data into its respective spatial component, which will be referred to as the pressure modes. The pressure modes are ranked according to their associated singular value in descending order. Figure 3 shows the four most prominent pressure modes.

After the pressure modes were generated, four FUN3D simulations were performed for each structural mode. In every simulation, the structure was deformed by applying a step input to one structural mode while the remaining structural modes were held fixed. The response, pressure distribution $P[n]$, was collected for the four previously mentioned simulations and $\left(P[n]-P_{0}\right)$ was projected onto the first eight (ranked) pressure modes resulting in eight generalized coordinates for every simulation.

The generalized coordinates are considered to be the system's response to the step inputs. However, in order to build a state-space model using the ERA, we need to derive the impulse response. Equation 15 shows how the impulse response relates to the step response:

$$
h_{t}=s_{t}-s_{t-1}, \quad h_{0}=s_{0},
$$

where $h_{i}$ and $s_{i}$ are the impulse response and the step response at time step $t$. After the impulse responses are derived from the step responses, we apply the ERA algorithm. The result of the procedure is a state space model that relates the generalized coordinates of the structural modes (the inputs) to the generalized coordinates of the pressure modes (the outputs).

This procedure is then repeated for different Mach numbers. In this paper we compare the accuracy of two models obtained for Mach 0.8 and 1.1. Each ROM is used to predict the pressure distribution over the wing under any structural deformation at the respective Mach number.

\section{IV.A. ROM Robustness}

In order to test the robustness of the generated ROM, the pressure distribution predicted by the ROM needs to be compared to the pressure distribution obtained with a high fidelity simulation for arbitrary inputs. We also would like to verify that the models generated are well suited for subsonic and supersonic freestream Mach numbers. The ROM and the FUN3D model simulations are both performed by deforming the structure, 


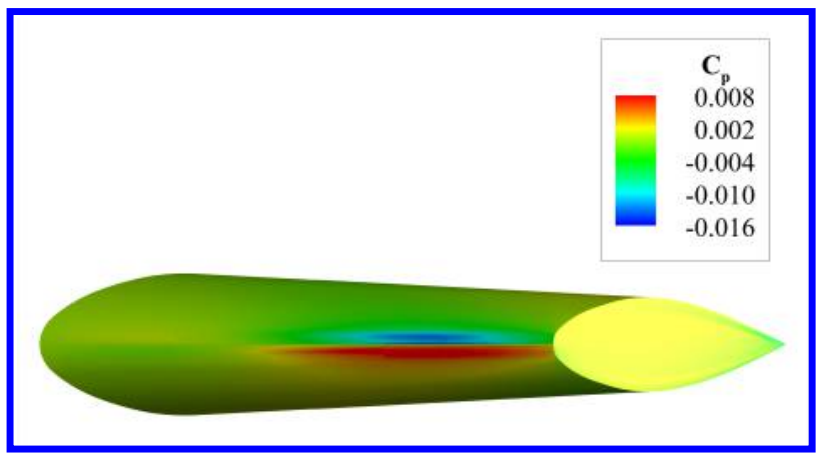

(a) First pressure mode.

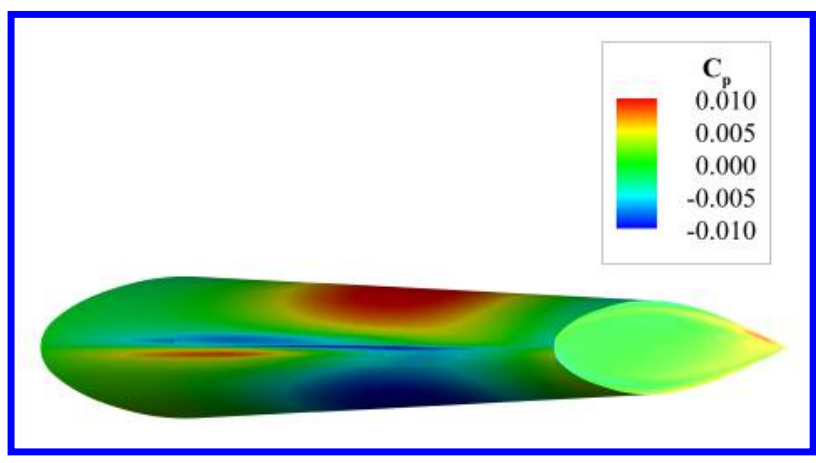

(c) Third pressure mode.

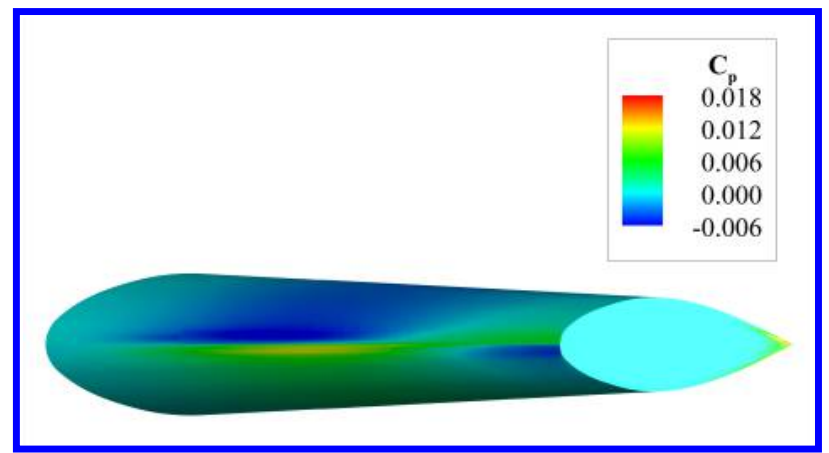

(b) Second pressure mode.

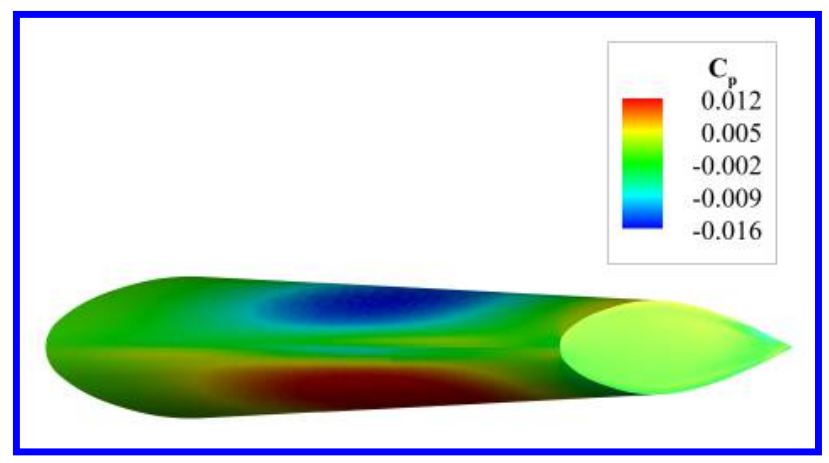

(d) Fourth pressure mode.

Figure 3. The first four pressure modes of the AGARD 445.6 wing. Note that the AGARD 445.6 wing is a very thin airfoil and the figure is not to scale, a visual distortion is applied in the z-direction for the aid of visualization.

and the predicted pressure distributions of both models are compared. The generalized coordinates of the structural modes serve as an input to both models. The predicted pressure distribution via the ROM is computed as follows

$$
\begin{gathered}
x[n+1]=A x[n]+B u[n], \quad x[0]=0, \\
\hat{P}[n]=C x[n]+D u[n], \\
P_{\mathrm{ROM}}[n]=P_{0}+\Phi \hat{P}[n] .
\end{gathered}
$$

Figure 4 shows the inputs, i.e., the structural deformations, applied to both the ROM and the FUN3D model for the Mach 0.8 and 1.1 cases.

The pressure distribution from the FUN3D simulation is then projected onto the same pressure modes used to generate the ROM for comparison. The generalized pressure coordinates of each model are plotted in Figure 6 and 7 for comparison. As the plots suggest, the ROM is able to accurately track the generalized coordinates associated with pressure mode 1 and 2 , however, the dynamics associated with some of the lower ranked pressure modes, e.g., pressure mode 3 , are not captured as well.

In order to properly demonstrate the effectiveness of the method in capturing the pressure distribution over the wing at the different Mach numbers, it is necessary to show the error associated in predicting the pressure distribution under various structural deformations. Since there are 50,827 pressure values for every time step, we compute the relative error as follows

$$
E[n]=\frac{\left|P_{\mathrm{ROM}}[n]-P_{\mathrm{FUN} 3 \mathrm{D}}[n]\right|}{\left|P_{\mathrm{FUN} 3 \mathrm{D}}[n]\right|} \times 100 .
$$

Figure 5 is a top view of $E[310]$ overlaid over the AGARD wing, the error in predicting the pressure distribution as computed by Equation 19 for $n=310$ at Mach 0.8 . 


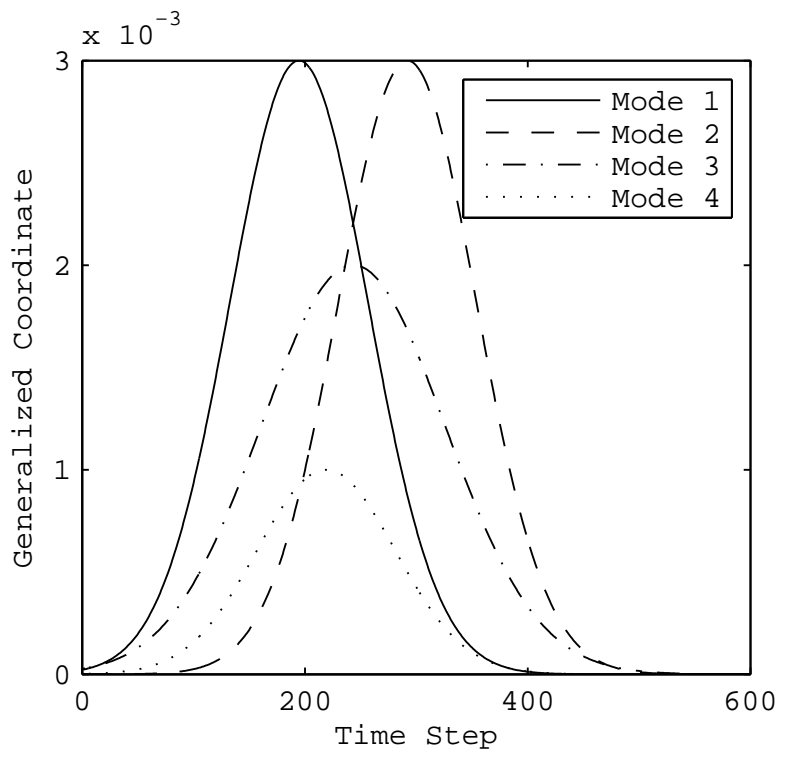

(a) Structural deformation for Mach 0.8 .

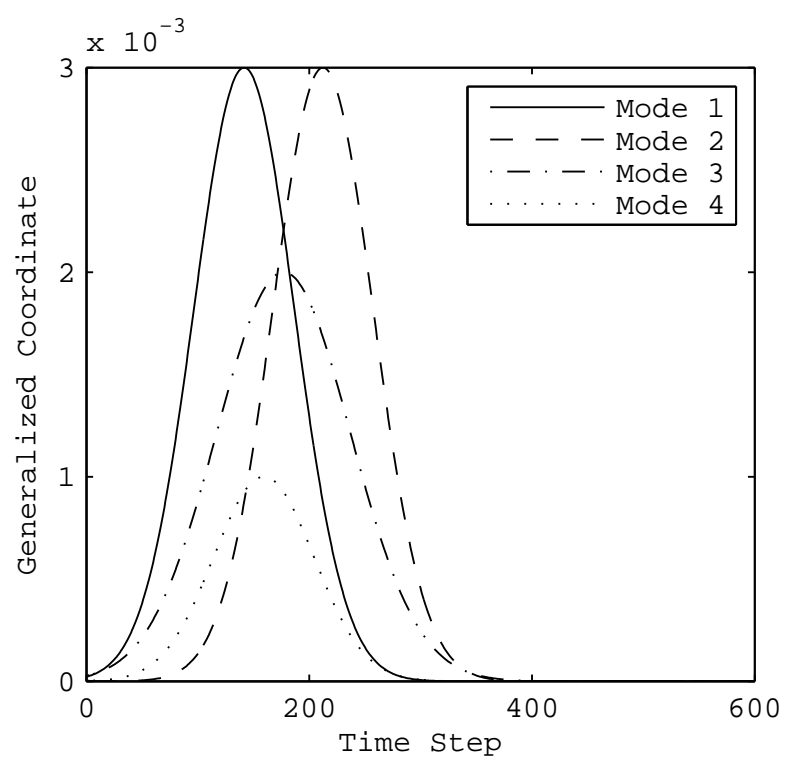

(b) Structural deformation for Mach 1.1.

Figure 4. Time history of generalized coordinates for each structural mode. The coordinates will serve as an input to the high fidelity aerodynamic model (FUN3D) and the reduced aerodynamic model (ROM).

The time step, $n=310$, is specifically chosen because the average error and its standard deviation are at their peak as can be seen by Figure 8(a). The average of the relative error and the associated standard deviation over the wing is computed and plotted in Figure 8 for both Mach 0.8 and Mach 1.1 when using only eight pressure modes.

The ROM is able to capture and recreate the pressure distribution over the AGARD wing to a very high accuracy, namely under $0.5 \%$ average error over the wing throughout the simulation. When the singular values of the pressure modes are compared, we observe that the singular values associated with the first four modes are $2.96 \times 10^{4}, 0.759$, 0.0455 and 0.0103 , respectively. This explains why

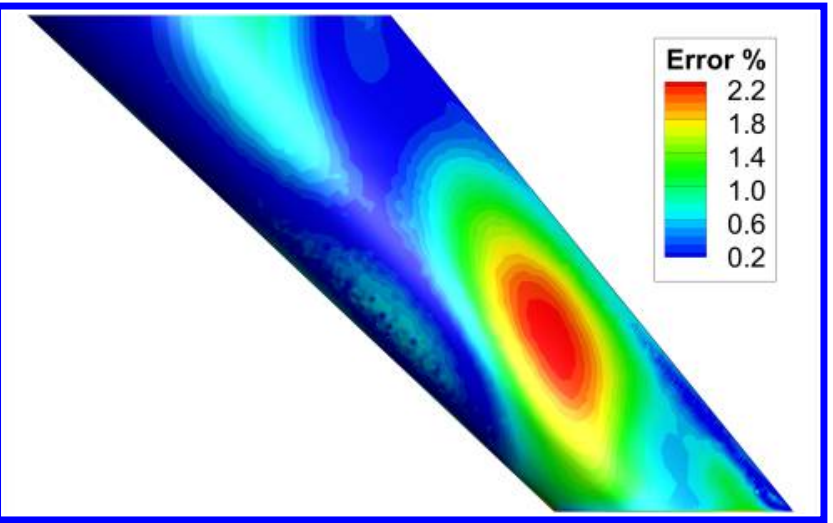

Figure 5. Relative error of predicted pressure (ROM) at time step 310 overlaid over the AGARD wing at Mach 0.8. Relative error is computed as defined by Equation 19. the ROM is still very accurate in reproducing the overall pressure distribution over the wing despite its poor predicting capability associated with some of the modes, such as mode 3, which has a relatively low singular value compared to modes 1 and 2 . The magnitude of the singular value reflects the importance of the associated pressure mode in contributing to the final pressure distribution.

Generating a better set of pressure modes means having better predictive capability for all modes. For example, it is clear that the dynamic behavior of the third pressure mode was not properly captured by the ROM. This can probably be improved by experimenting with applying the POD method on different datasets. Such datasets could be varied by adjusting the magnitude of the inputs applied to structural modes or changing the time step of the CFD simulation.

Another area for improving the ROM is looking into different system identification techniques. The ERA performed well, but different algorithms could be compared as they might capture richer dynamics or different interactions between the structural modes and pressure modes. 


\section{Conclusion}

The results confirm that the proposed method for generating the ROM is capable of accurately predicting the unsteady pressure distribution over the AGARD wing when the structure is arbitrarily deformed. Furthermore, the ROM is capable of efficiently simulating the pressure distribution for hundreds of time steps while FUN3D requires many computational hours for the same simulation. It is worth mentioning that the ROM is only valid under conditions that were used to generate the CFD data; i.e. Mach number, angle of attack, etc.

While it was demonstrated that only a small subset of the POD pressure modes, namely eight, can capture the unsteady pressure response over the entire wing with high accuracy at subsonic and supersonic flow conditions, there is still room for improvement in increasing the accuracy of the ROM. Ongoing work continues, investigating methods that can provide better POD modes that capture the dynamics between the unsteady pressures and the structural response.

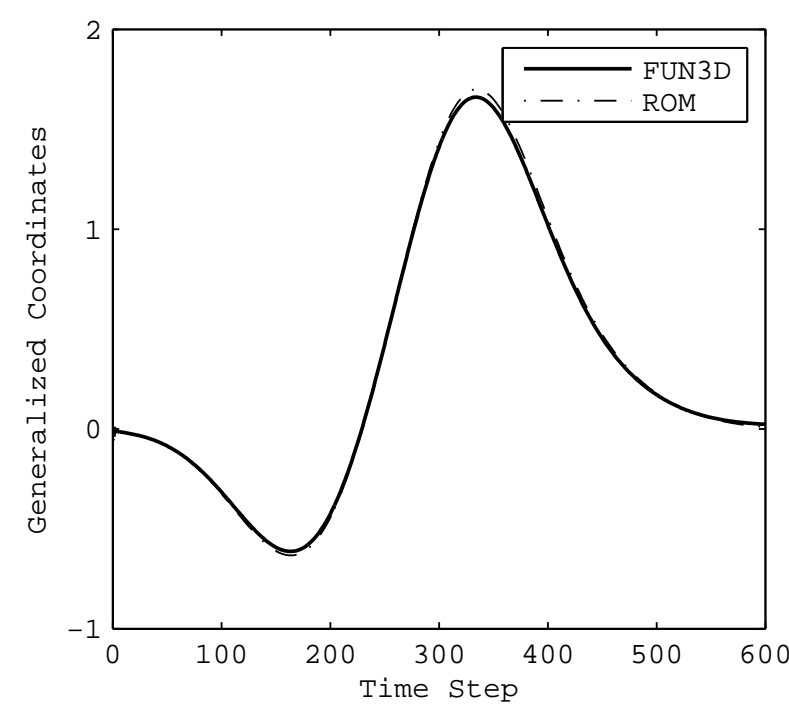

(a) Time history of generalized pressure coordinates associated with the first pressure mode.

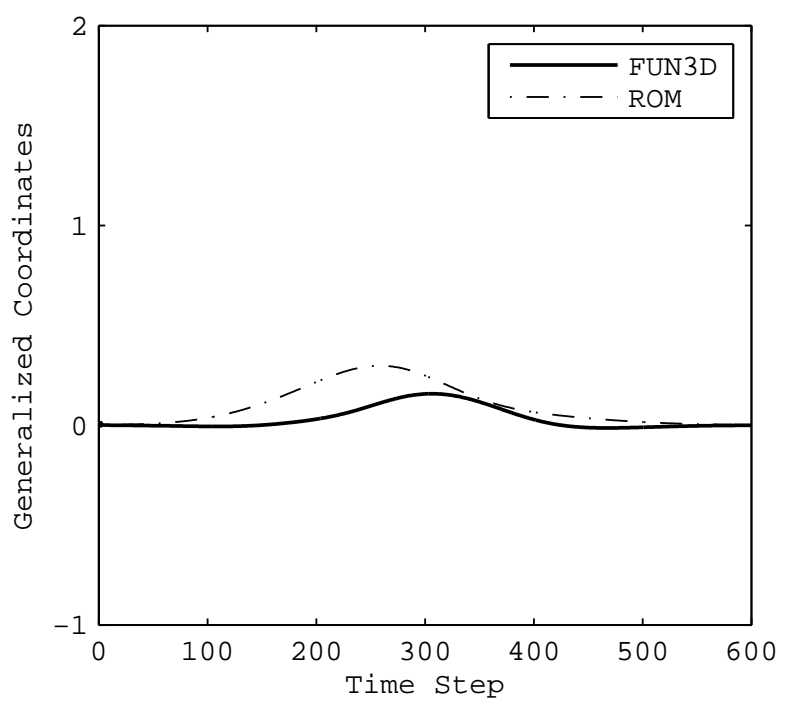

(c) Time history of generalized pressure coordinates associated with the third pressure mode.

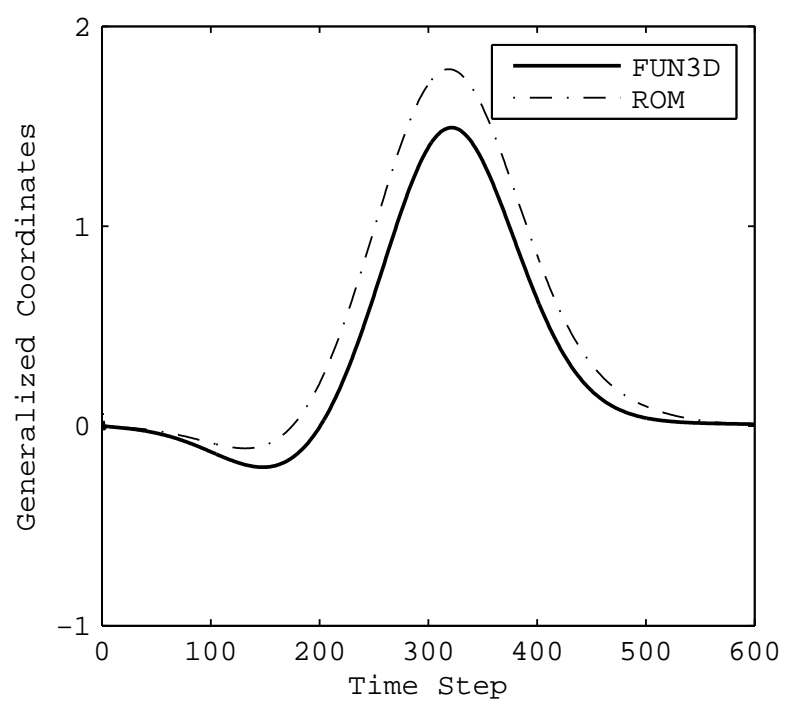

(b) Time history of generalized pressure coordinates associated with the second pressure mode.

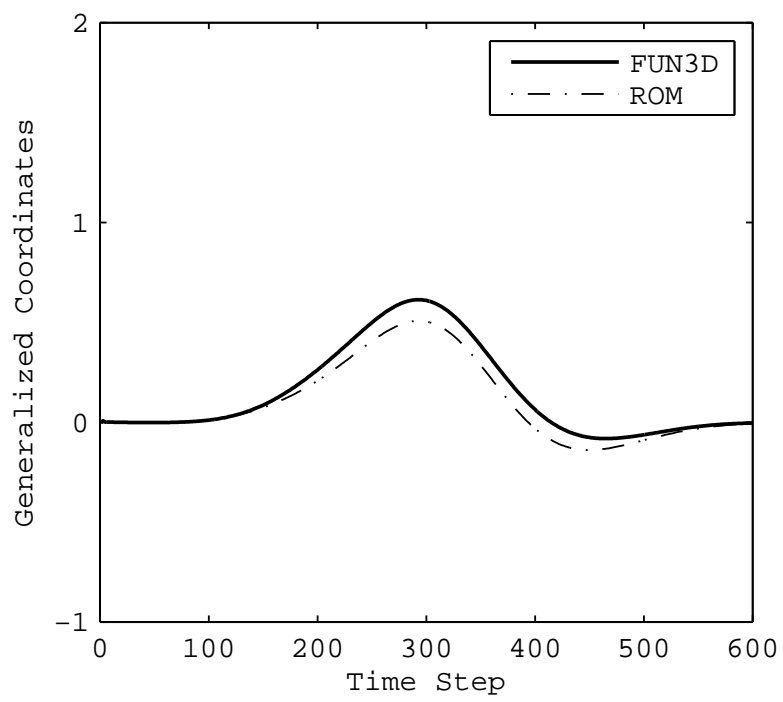

(d) Time history of generalized pressure coordinates associated with the fourth pressure mode.

Figure 6. Reduced order model compared to output from FUN3D at Mach 0.8. 


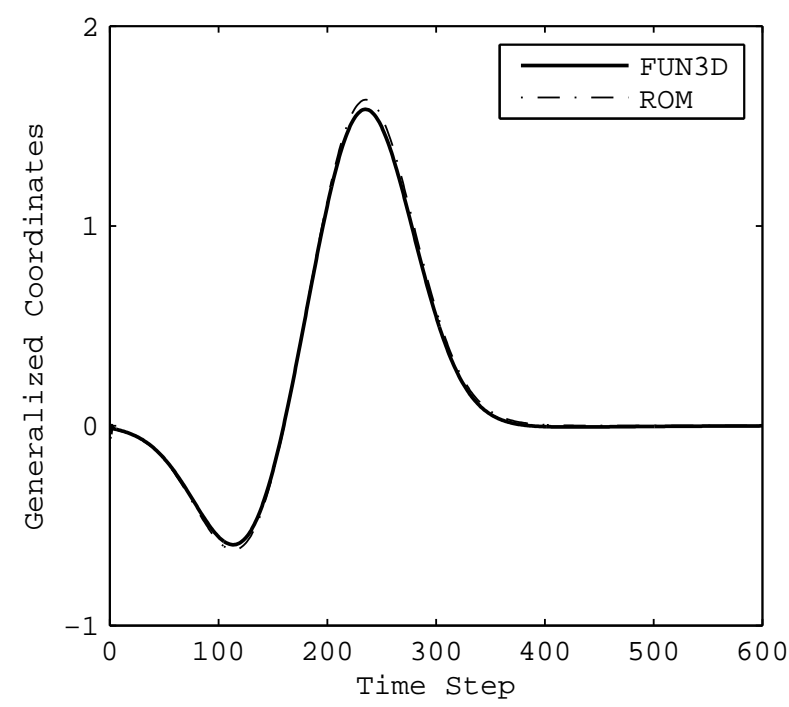

(a) Time history of generalized pressure coordinates associated with the first pressure mode.

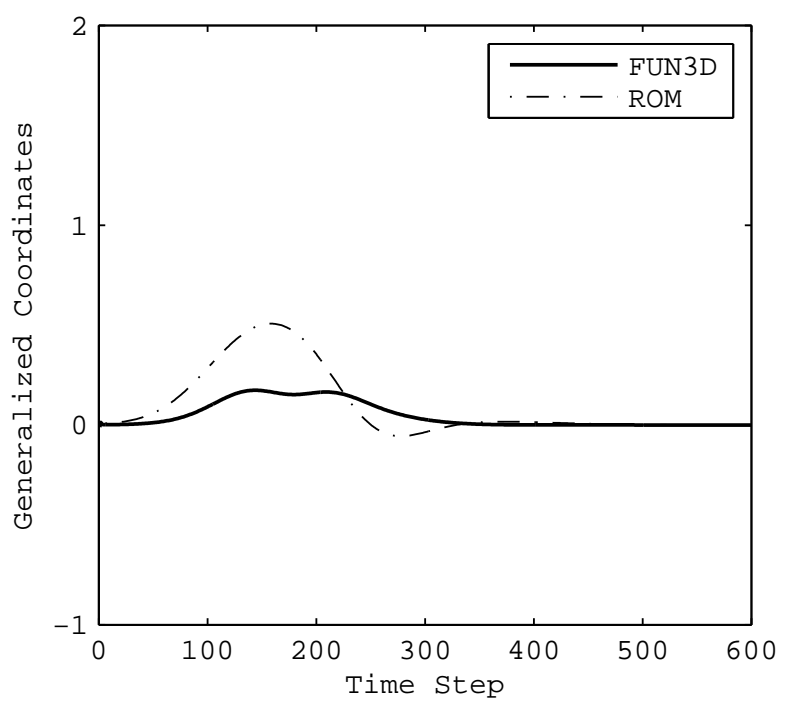

(c) Time history of generalized pressure coordinates associated with the third pressure mode.

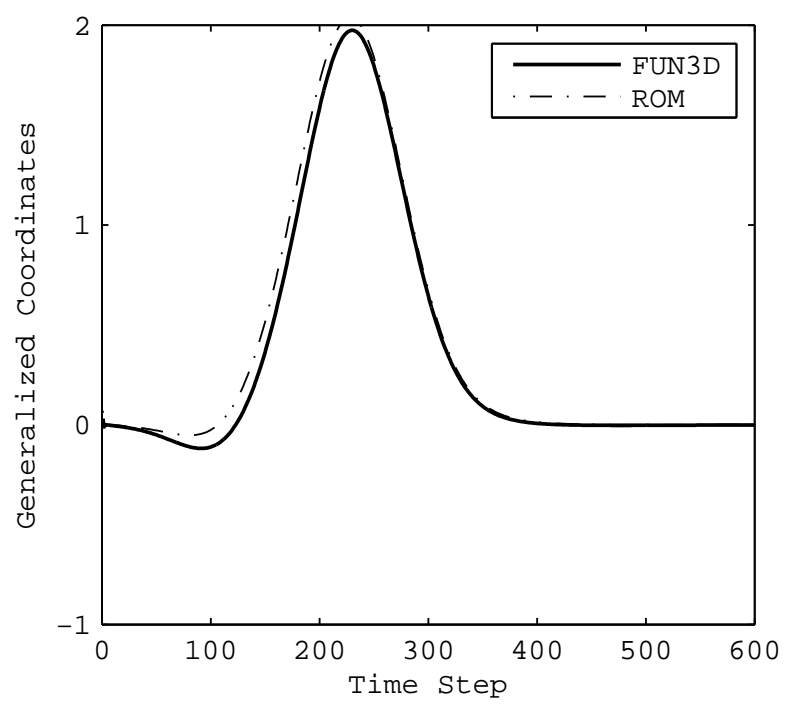

(b) Time history of generalized pressure coordinates associated with the second pressure mode.

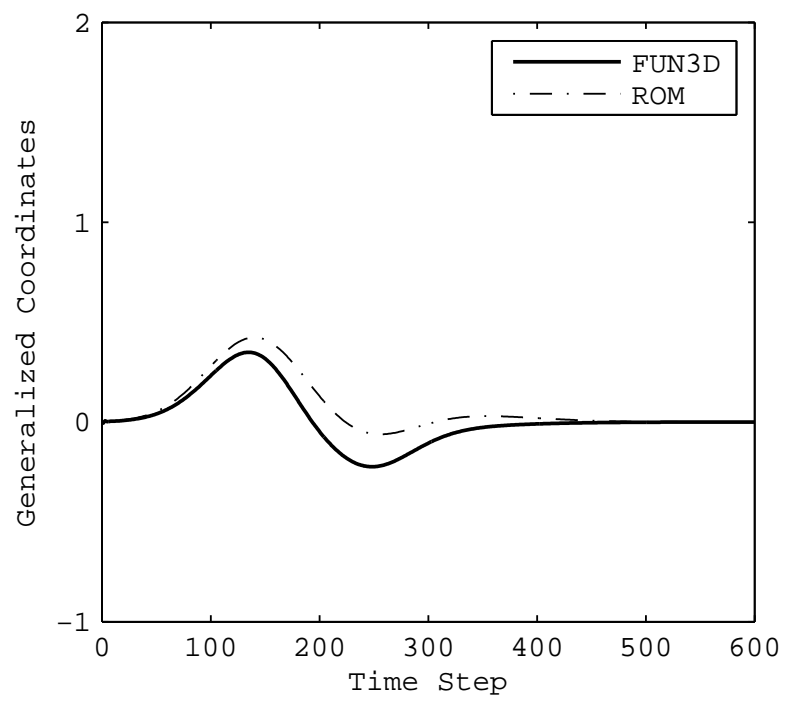

(d) Time history of generalized pressure coordinates associated with the fourth pressure mode.

Figure 7. Reduced order model compared to output from FUN3D at Mach 1.1. 


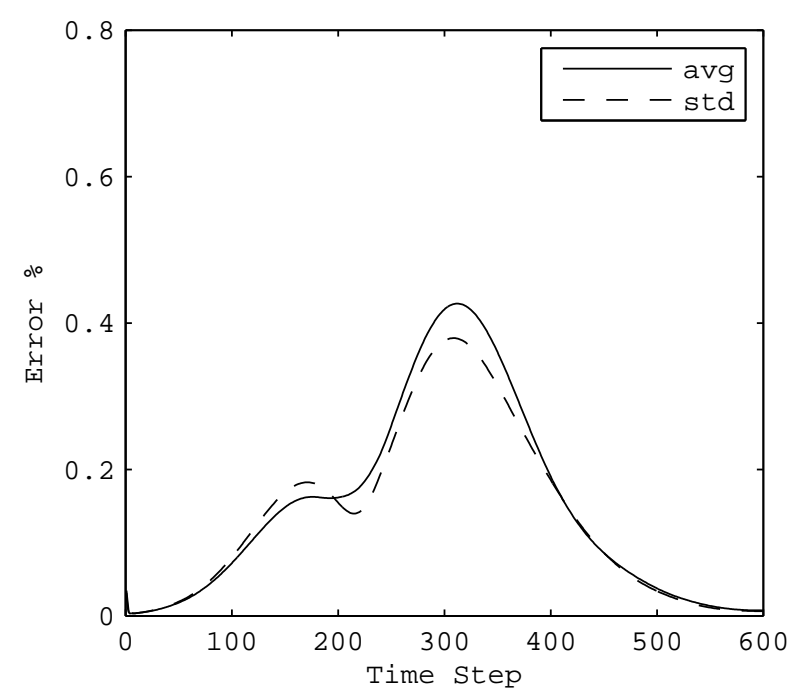

(a) Average and standard deviation of the percent error over the AGARD wing at every time step for Mach 0.8 .

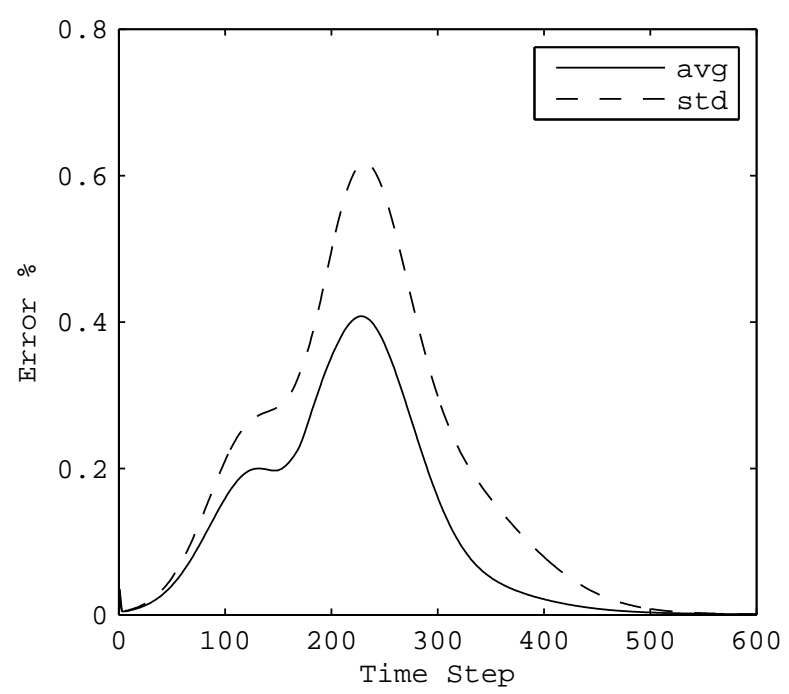

(b) Average and standard deviation of the percent error over the AGARD wing at every time step for Mach 1.1.

Figure 8. Comparison of the ROM to the high fidelity CFD solution.

\section{References}

${ }^{1}$ Volterra, V., Theory of Functionals and of Integral and Integro-Differential Equations, Dover, New York, 1959.

${ }^{2}$ Loeve, M., Functions Aleatoire de Second Ordre, C. R. Academie des Sciences, Paris, 1945.

${ }^{3}$ Karhunen, K., Zur Spektral Theorie Stochastischer Prozesse, Ann. Acad. Sci. Fennicae, Ser, 1946.

${ }^{4}$ Berkooz, G., Holmes, P., and Lumley, J. L., "The Proper Orthogonal Decomposition in the Analysis of Turbulent Flows," Annual Reviews of Fluid Mechanics, Vol. 25, 1993, pp. 539-575.

${ }^{5}$ Silva, W. A., Discrete-Time Linear and Nonlinear Aerodynamic Impulse Responses for Effecient CFD Analyses, Ph.D. thesis, College of William and Mary, 1997.

${ }^{6}$ Silva, W. A., "Reduced-Order Models Based on Linear and Nonlinear Aerodynamic Impulse Responses," CEAS/AIAA/ICASE/NASA Langley International Forum on Aeroelasticity and Structural Dynamics, 1999, pp. 369-379.

${ }^{7}$ Raveh, D., Levy, Y., and Karpel, M., "Aircraft Aeroelastic Analysis and Design Using CFD-Based Unsteady Loads," $A I A A, 2000$.

8 Beran, P. S. and Pettit, C. L., "Prediction of Nonlinear Panel Response Using Proper Orthogonal Decomposition," AIAA, 2001.

${ }^{9}$ Chen, H., Reuss, D. L., Hung, D. L., and Sick, V., "A practical guide for using proper orthogonal decomposition in engine research," International Journal of Engine Research, Vol. 14, No. 4, 2013, pp. 307-319.

${ }^{10}$ Juang, J.-N. and Pappa, R. S., "An Eigensystem Realization Algorithm for Modal Parameter Identification and Model Reduction," J. Guidance, Vol. 8, No. 5, 1985, pp. 620-627. 